\title{
Efficacy of optical coherence tomography and fundus fluorescein angiography in detection of diabetic macular edema
}

\author{
Veshal Madan ${ }^{1}$, Renu Magdum ${ }^{2, *}$, Somnath Ghosh ${ }^{3}$ \\ ${ }^{1}$ Director in-charge and Associate Professor, ${ }^{2}$ Professor and HOD, Dept. of Ophthalmology, Dr. D Y Patil Medical College and \\ Hospital, Pimpri, Pune, Maharashtra, ${ }^{3}$ Associate Professor, Dept. of Ophthalmology, Dr DY Patil Institute of Optometry \& Visual \\ Sciences, Pimpri, Pune, Maharashtra, India
}

*Corresponding Author:

Email: renumagdum@yahoo.co.in

\begin{abstract}
Purpose: Significance of clinical DME in an up becoming capital nation of diabetes mellitus in world has socio-economic effect due to VTDR is identified as the major cause of irreversible blindness in DR subjects in all age groups. Therefore, the need for reliable, safe and immediate detection of DME is a need for prevention of the disease \& related socio economic burden. The study was to compare the diagnostic potential of two commonly established diagnostic instruments i.e.-SD-OCT \& FFA for the detection of DME.

Materials and Methods: A prospective study with purposively sampling technique was carried on 308 eyes of 154 type II DM patients to compare the detection potential between two popular retinal diagnostic techniques, SD-OCT a non-invasive technique versus FFA invasive method for DME. The standard parameters such as focal macular edema, diffuse macular edema and mixed edema with cystoid type were evaluated with FFA. Subsequently DME classification with presence of intraretinal edema, sub retinal fluid and mixed edema were diagnosed with SD-OCT for the same pool of patients. Clinical features in DME using reference 'EDTRS' scale of measurement.

Results: Noninvasively 305 (99.1\%) eyes were detected positive for various type of DME by SD-OCT while 282 (91.6\%) eyes were detected positive by FFA. The prevalence of different types of DME such as cystoids, diffuse, focal and mixed was $7.8 \%$, $13 \%, 7.1 \%$ and $63.7 \%$ respectively as diagnosed with FFA. Whereas, SRF, IR edema and mixed type was $4.9 \%, 3.6 \%$ and $90.6 \%$ respectively as diagnosed with SD-OCT. DME was not detected by FFA in $8.4 \%$ of eyes in overall study population. Whereas, SD-OCT was unable to detect DME in $0.9 \%$ of eyes. In our study overall performance of SD-OCT was better in detection of DME when compared to FFA.

Conclusion: The present study showed the diagnostic potential and efficacy of SD-OCT over FFA in reliable and safe detection of DME in Type II Diabetic Mellitus patients was more and was easy and comparable to diagnostic findings of FFA specifically in cases of sub retinal macular as well choroidal edema due DME by SD-OCT.
\end{abstract}

Keywords: Diabetic macular edema, Fundus fluorescein angiography, Optical coherence topography.

\section{Introduction}

Diabetic Macular Edema (DME) is the most common cause of moderate loss of vision ${ }^{1}$ which needs to detect as early for effective management of visual loss. Fundus Fluorescein Angiography (FFA) uses invasive mode of investigative technique to assess the retinal choroidal flow to locate any leakage, block or neovascularisation in arterio-venous architecture and plexus in and around macular region with incidence of fatal anaphylaxis. ${ }^{2}$ FFA indicates areas of retina with scanty blood flow through the macular capillaries as relative hypo fluorescent. The need for non invasive procedure like improvised OCT techniques enable deeper and faster analysis and documentation of the macular anatomy with no risk of drug related anaphylaxis or intravenous procedures.

FFA was considered gold standard for diagnoses of retinal vasculature diseases ${ }^{3}$ but in respect to macular involvement which has no blood supply hence the use of spectral domain optical coherence tomography (SDOCT) is with high resolution three dimensional imagery of the deep section of macular structure gives detailed disruption of macular structures due to DME with relation to its affection on visual acuity. ${ }^{4} \mathrm{SD}-\mathrm{OCT}$ is used extensively for clinical findings which can be predictive for early detection of diabetic macular changes deep and intermediate layers of retina for management which very much comparable to FFA and stereo fundus photography hence new techniques for quantitatively and qualitatively measuring retinal thickness are challenging the old trusted techniques. ${ }^{5}$

The purpose of the present study was to observe the comparative performance of Fundus Fluorescein Angiography (FFA) and Spectral Domain Optical Coherence Tomography (SD-OCT) in detection of Diabetic Macular Edema (DME).

\section{Materials and Methods}

This prospective study was conducted in Department of Ophthalmology, Dr DY Patil Medical College, Hospital \& Research Centre, Pimpri, Pune during the period April 2014 and March 2016 after obtaining clearance from Ethical Committee. 308 eyes with Diabetic Macular Oedema (DME) patients having were recruited in the study. The recruitment of the patients was done based on the presets inclusion and exclusion criteria. Patients aged over 40 years with diabetic retinopathy along with clinically significant 
macular oedema were recruited for the study. Patients with diseases in ocular media and complications in retina apart from the diabetic retinopathy were excluded from the study. Recruited patients were underwent complete ophthalmological examination. The recruited study patients were underwent Fundus Fluorescein Angiography (FFA) and Spectral Domain Optical Coherent Topography (SD-OCT) respectively.

Fundus Fluorescein Angiography: Fluorescein angiography (FA) has played important roles in the investigation of patients with DR. Areas of capillary nonperfusion are clearly visualized, as is enlargement of the foveal avascular zone (FAZ). In addition, FA demonstrates active leakage from micro aneurysms in DME and is also helpful in confirming leakage from suspicious areas of neovascularization. Conventional FAs typically cover $30-55^{\circ}$ of the retina. While standard fundus cameras can be steered to image different parts of the retina, the various images are captured at different phases of the angiogram. As a result of the short transit time for fluorescein, important information may be lost. Fluorescein angiography (FA) has been an important method to evaluate DME and although not considered a screening exam, it provides important information about retinal perfusion, bloodretinal barrier integrity, and new vessel growth. Angiographic classifications of DME are focal or diffuse DME. Focal macular edema is characterized by the presence of localized areas of retinal thickening associated with focal leakage of individual microaneurysms or clusters of microaneurysms or dilated capillaries. Diffuse macular edema is a more generalized and chronic form.

The use of ophthalmic instrument inventor company Carl Zeiss FF 450 plus fundus fluorescein angiogram model is used for detection and tracking the retinal choroidal vascular flow in diabetic retinopathy and ischemic retinopathy conditions. The role of FFA is to detect early areas of ischemia and areas of capillary non perfusion in and around the macular region in cases of reduced visual acuity in known DR subjects. The FFA image reflects the ischemic diabetic retinopathy and areas of Para macular region.

Spectral domain Optical Coherence Angiography (SD-OCT): Spectral Domain-OCT is a diagnostic imaging technology that provides anatomical section of biological tissues. OCT imaging is analogous to B-scan ultrasonography with enhancement to a sub tissue level, except that OCT measures light rather than acoustic waves.OCT is working principle is by measuring the echo delay with intensity of backscattered light from the internal tissue microstructures and because the echo time delays of light are too fast to measure directly, an optical correlation technique, known as Michelson low coherence interferometry is used. Low-coherence light from a super luminescent diode (SLD) is directed through a beam splitter and is segregated into a sample beam that is focused onto the patient's retina and a reference beam which travels a calibrated delay path. Normally near infrared $843 \mathrm{~nm}$ light backscattered by retinal structures interferes with light from the reference beam and the interference of the echoesis detected to measure the back scattering signal versus delay or depth.

Standard operative procedure was followed and the investigations were done by the ophthalmologist. DME was classified based on the EDTRS classification. ${ }^{5}$ Clinical features in DME using reference 'EDTRS' scale of measurement. Sub classification by presence of sub retinal fluid and intra retinal edema were the hallmark findings of SD-OCT while detection of areas of non- perfusion \& hyper perfusion due subsequent neovascularisation in case of ischemic macular edema were only detected by FFA.

\section{Data Analysis}

Data was analyzed using Microsoft Excel and SPSS (version 17.0). Qualitative data was summarized in percentages. Quantitative data was summarized as mean and standard deviations. Appropriate statistical tests such as Z-test, significance of correlation coefficient were applied for quantitative data. Significance level was kept at $5 \%(\mathrm{p}<0.05)$.

\section{Results}

Three hundred and eight eyes of 154 patients with Diabetic Macular Edema (DME) were analysed in the present study. The age of the patient ranged from 40 years to 76 years with the average of 60.64 years. The maximum number of patients (90) was from the age group 65-69 years followed by 60-64 years (56) (Fig. 1). Nearly $89 \%$ patients were above 50 years. It has also found that out of 154 patients about $70.13 \%$ were male and rest $29.87 \%$ was females.

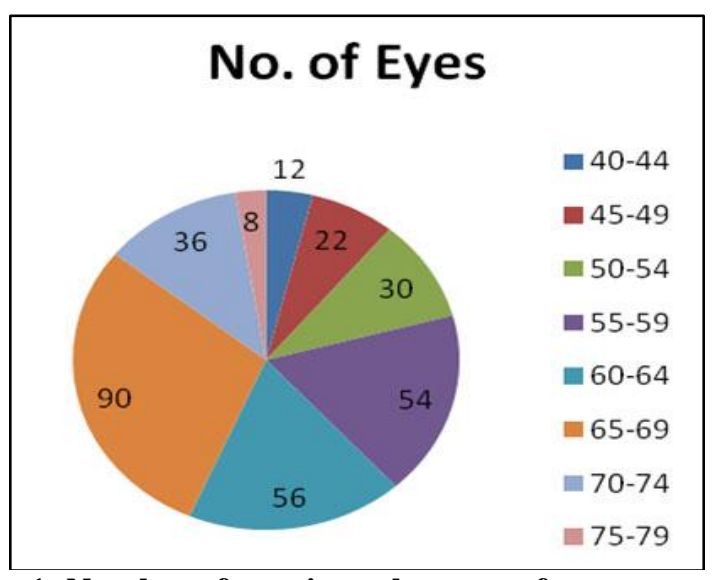

Fig. 1: Number of eyes in each group of ages

Visual status was observed among all the patients. It has been observed that major portion of eyes consisting of $48.7 \%$ were from vision grade three $(6 / 24)$ category, followed by grade four (6/18) which contribute to $35.4 \%$. Remaining eyes in various grades 
were having individual share less than $10 \%$ of total eyes examined (Fig. 2).

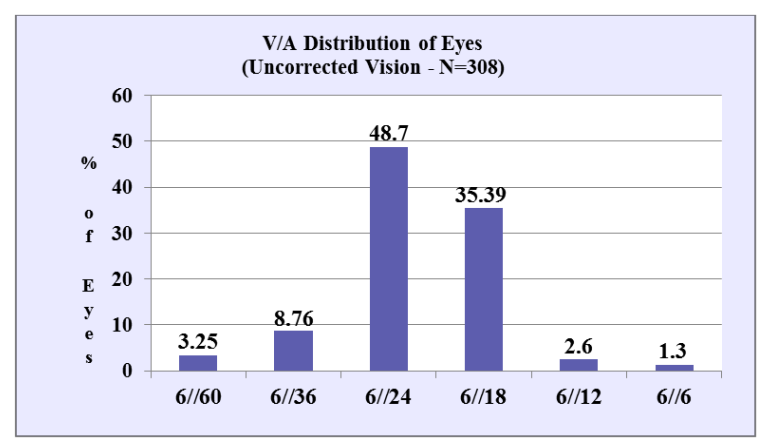

Fig. 2: Visual outcomes of patients with diabetic macular edema

All the patients underwent through Fundus Fluorescein Angiography (FFA) to detect Diabetic Macular Edema (DME). Out of the total 308 eyes, DME was detected in 282 eyes $(91.55 \%)$ with FFA. However, DME could not be detected in 26 eyes $(8.45 \%)$ with FFA.

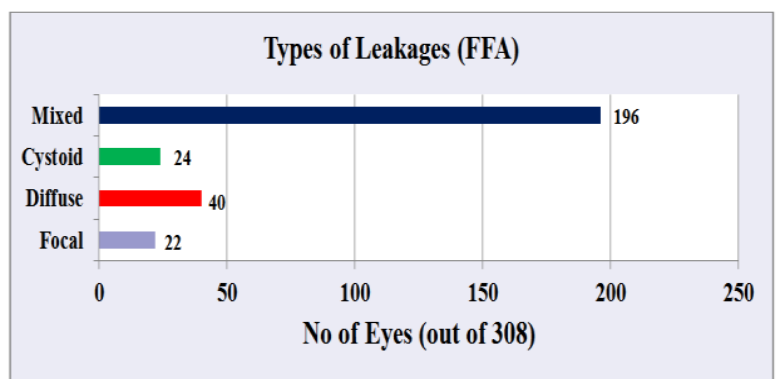

Fig. 3: Detection of different types of diabetic macular edema through FFA

The DME was also classified based their clinical features. Out of the 282 eyes, Focal Macular Edema detected in $22(7.14 \%)$ eyes, while $11(3.57 \%)$ eyes only intra retinal edema not involving the Macula. Mixed Macular Edema was detected in 196 eyes on FFA. Cystoids changes were commonly seen in diffused DME only (Fig. 3).

On the other hand, SD-OCT could detect DME in 305 eyes out of 308 eyes (99.02\%), which was higher compare to detection through FFA. Mixed edema with foveal involvement and obstruction of fluid was detected in eyes in 279 eyes $(90.50 \%)$ out 308 eyes. Intra retinal edema was detected on $11(3.57 \%)$ eyes out 308 eyes and sub retinal fluid was 15 (4.8\%) eyes detected through OCT which were completely missed out on FFA (Fig. 4).

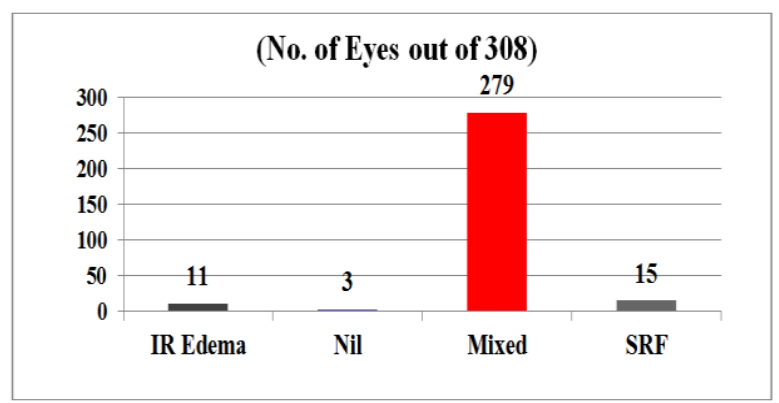

Fig. 4: Detection of different types of diabetic macular edema through SD-OCT

When the figures of number of eyes are compared, it shows that SD-OCT is found more effective to detect abnormalities than FFA. FFA could not detect 26 eyes with abnormality while SD-OCT could not detect only 3 eyes with abnormality. On FFA, focal leak without macular involvement was seen in 22 eyes out of which 11 eyes did show macular involvement on OCT. FFA also missed eyes with SRF (neurosensory detachment only).

The kappa statistic was used to find out the agreement between two instruments. The kappa coefficient is widely used statistic for measuring the degree of reliability between two raters. These ratings are usually categorized as "Yes" or "No", based on how much each rater would answer a specific question about the record. The results of these responses are generally placed into a $2 \times 2$ table. In the present case one rater is FFA technique and another being OCT. The detection of DME is a record under study. The values of kappa range from -1 to +1 , with -1 indicating perfect disagreement and +1 indicating perfect agreement between the two raters. In the present study, the agreement between two instruments was found $\mathrm{K}=$ 0.19243; which was good strength of agreement.

\section{Discussion}

The prevalence of Diabetic Macular Edema (DME) increases with age as $50.6 \%$ were in $61-70$ years age group which correlate to previous studies. ${ }^{6-8}$ The present study was carried out to determine the potential diagnostic tool between FFA and SD-OCT. The study showed promising results by both FFA and SD-OCT. Based on the present study findings, FFA could detect 4 types of diabetic macular oedema namely, Mixed, Cystoid, Diffuse and Focal. While Optical Coherence Tomography detected 3 types of diabetic macular oedema namely, intraretinal edema, sub retinal fluid and mixed macular oedema from the same pool of patients.

In the present study, FFA detected 196 mixed macular edema where as OCT detected 279 mixed macular edema. Cystoid, diffuse and focal were 24, 40 and 22 detected by FFA respectively. While, OCT detected 11 eyes with IR Edema and 15 eyes with SRF. Antcliff et al. in 2000 have conducted a study on 
"compare optical coherence tomography (OCT) with fundus fluorescein angiography (FFA) for the detection of cystoids macular edema (CME) in patients with Uveitis" and found that OCT performs better compared to FFA in terms of detection of cystoid macular edema. They have also mentioned that sensitivity for detecting CME was 96\% (including the eyes with sub-retinal fluid) and the OCT specificity was $100 \% .^{5}$

In the present study, SRF was detected with OCT for 15 eyes whereas, FFA could not detected the same for the same pool of patients. A similar finding was observed by Antcliff et al. 2000. ${ }^{5}$

Detection of macular edema was higher in number with OCT compared to FFA in the present study. The FFA detected 220 eyes with DME where OCT detected 305 eyes with DME.

In 2002, Strom et al. have reported that the degree of agreement between subjectively and objectively assessed retinal thickenings was very good implying that changes in diabetic macular edema can be detected accurately with OCT. ${ }^{9}$

In 2012, Maalej et al. have reported about the importance of OCT in routine clinical assessment of diabetic macular oedema. They have also stated that identifying the structural changes in eyes with DME using OCT may allow more effective management of DME patients. ${ }^{10}$

Shoughy et al. 2016, have done a study on "selective and complementary use of optical coherence tomography and fluorescein angiography in retinal practice" and stated that the OCT provides detailed imaging of anatomical retinal layers, allows detection of micro-structural changes and helps perform quantitative assessment during follow up and FFA is used to evaluate retinal vascular perfusion and integrity of the inner blood-retinal barrier. ${ }^{11}$

FFA is known to be a sensitive method for qualitative assessment of fluid leakage in diabetic macular edema; FFA is an invasive procedure, with side effects ranging from nausea to its rare complication of anaphylaxis and death. While, OCT is non-invasive, comfortable, safe, and fast and can be repeated as often as is required and offers an alternative to FFA in the follow-up of changes in retinal thickness after laser photocoagulation and intravitreal steroid injections.

\section{Conclusion}

Detection of diabetic macular oedema and management is important to avoid the vision loss. Therefore need to compare the efficacy of two diagnostic modalities, SD-OCT and FFA to detect macular oedema due to diabetic mellitus. There is also need for safe and effective patient friendly method for detection of diabetic macular oedema. In the present study, both FFA and SD-OCT have shown good potential to detect the diabetic macular oedema.
However, overall performance in-terms of safety and detection ability of SD-OCT were comparatively better in compared to FFA.

\section{References}

1. Sen S, Chakraborty R, De B. Complications of Diabetes Mellitus. Diabetes Mellitus in 21st Century, Page No. 69100.

2. Singh R, Ramasamy K, Abraham C, Gupta V, Gupta A. Diabetic retinopathy: An update. Indian J Ophthalmol. 2008;56(3):179-188.

3. Hajar S, Hazmi AA, Wasli, M, Mousa A, Rabiu M. Prevalence and causes of blindness and diabetic retinopathy in Southern Saudi Arabia. Saudi Med J. 2015;36(4):449-455.

4. B Askira, B Mubi. Blindness in Patients with Diabetes Mellitus Attending a Diabetes Clinic at the University of Maiduguri Teaching Hospital, Maiduguri. The Internet Journal of Ophthalmology and Visual Science. 2012 Volume 9 Number 1.

5. Antcliff RJ, Stanford MR, Chauhan DS, Graham EM, Spalton DJ, Shilling JS, fytche TJ, Marshall J. Comparison between optical coherence tomography and fundus fluorescein angiography for the detection of cystoid macular edema in patients with uveitis. Ophthalmology. 2000;107(3):593-9.

6. Lee R, Wong TY, Sabanayagam C. Epidemiology of diabetic retinopathy, diabetic macular edema and related vision loss. Eye Vis (Lond). 2015;2:17. doi:10.1186/s40662-015-0026-2. eCollection 2015.

7. Varma R, Bressler NM, Doan QV, Gleeson M, Danese M, Bower JK, Selvin E, Dolan C, Fine J, Colman S, Turpcu A. Prevalence of and Risk Factors for Diabetic Macular Edema in the United States. JAMA Ophthalmol. 2014;132(11):1334-1340.

8. Kaveeshwar SA, Cornwall J. The current state of diabetes mellitus in India. The Australasian Medical Journal. 2014;7(1):45-48. doi:10.4066/AMJ.2013.1979.

9. Strøm C, Sander B, Larsen N, Larsen M, Lund-Andersen H. Diabetic macular edema assessed with optical coherence tomography and stereo fundus photography. Invest Ophthalmol Vis Sci. 2002;43(1):241-5.

10. Maalej A, Cheima W, Asma K, Riadh R, Salem G (2012) Optical Coherence Tomography for Diabetic Macular Edema: Early Diagnosis, Classification and Quantitative Assessment. J Clinic Experiment Ophthalmol S2:004. doi:10.4172/2155-9570.S2-004.

11. Shoughy SS, Kozak I. Selective and complementary use of Optical Coherence Tomography and Fluorescein Angiography in retinal practice. Eye Vis (Lond). 2016;3:26. doi:10.1186/s40662-016-0058-2. 